\title{
Effets des pratiques paysannes de fertilisation sur les caractéristiques chimiques d'un sol ferrugineux tropical lessivé en zone cotonnière à l'Ouest du Burkina Faso
}

\author{
Bernard BACYE ${ }^{1 *}$, Hyacinthe Sami KAMBIRE ${ }^{2}$ et Arsène Salibo SOME ${ }^{1}$ \\ ${ }^{1}$ Université Nazi BONI (UNB), Burkina Faso. \\ ${ }^{2}$ Institut de l'Environnement et de Recherches Agricoles (INERA), Burkina Faso. \\ "Auteur correspondant ; E-mail: bbacye@gmail.com; 01 BP 1091 Bobo-Dioulasso 01, Burkina Faso. \\ Tél : (+226) $20980635 /(+226) 70260300$.
}

\section{REMERCIEMENTS}

Les auteurs remercient vivement l'Université Nazi BONI (UNB) et l'Institut de l'Environnement et de Recherches Agricoles (INERA) pour leur contribution au financement de ce travail.

\section{RESUME}

La dégradation des sols cultivés constitue l'une des contraintes majeures limitant la productivité de l'agriculture en Afrique sub-saharienne. Cependant, dans une même zone climatique, ce sont surtout les pratiques agricoles qui vont déterminer le niveau de fertilité d'un sol donné. La présente étude, conduite en zone cotonnière à l'Ouest du Burkina Faso, avait pour objectif d'évaluer les effets des pratiques de fertilisation sur les caractéristiques chimiques d'un sol ferrugineux lessivé. Un échantillon de 22 champs a permis de caractériser les pratiques de fertilisation à l'aide d'enquêtes et de prélever l'horizon $0-20 \mathrm{~cm}$ du sol pour des analyses de laboratoire. Les principales pratiques de fertilisation identifiées sont les apports de fumier sur des portions des champs, les apports d'engrais sur les cultures de cotonnier et de maïs et la jachère. Les résultats ont montré que seules les apports organiques ont permis une amélioration significative au seuil de $5 \%$ des teneurs en matières organiques, de la capacité d'échange cationique (CEC), de la somme de bases échangeables et du $\mathrm{pH}$ du sol. Il apparait donc que l'utilisation durable des sols dans la zone nécessite le renforcement de la fumure organique à travers le développement de nouveaux modèles de restitutions organiques.

(c) 2019 International Formulae Group. All rights reserved.

Mots clés: Sol ferrugineux lessivé, caractéristiques chimiques, effets des pratiques de fertilisation, zone cotonnière, Ouest du Burkina Faso.

\section{Effects of farmers' fertilization practices on the chemical characteristics of a lixisol in cotton zone of Western Burkina Faso}

\begin{abstract}
The depletion of cultivated soils is one of the major constraints limiting the productivity of agriculture in sub-Saharan Africa. However, within a same climatic zone it is agricultural practices that will mostly determine the fertility level of a given soil. The objective of the current study conducted in the cotton
\end{abstract}


zone of Western Burkina Faso, was to evaluate the effects of farmers' fertilization practices on the chemical characteristics of a lixisol. A total number of 22 fields was sampled. The fertilization practices were characterized through surveys and soil sampling at the depth of 0-20 cm for laboratory analyses. The main fertilization practices identified are organic manure application on portions of fields, application of mineral fertilizers on cotton and maize crops, and fallowing. The results of soil analyses showed at 5\% error threshold that only organic manure application allowed a significant improvement of soil organic matter, soil Cationic Exchange Capacity (CEC), total exchangeable bases and the $\mathrm{pH}$ of the soil. It appears therefore that the sustainability of land use in the area requires strengthening the use of organic manure through the development and implementation of new models of organic-based soil replenishment.

(C) 2019 International Formulae Group. All rights reserved.

Keywords: Lixisol, chemical characteristics, effects of the fertilization practices, cotton zone, Western Burkina Faso.

\section{INTRODUCTION}

La faible fertilité des sols est considérée comme l'une des contraintes majeures limitant la productivité de l'agriculture subsaharienne (Bationo, 2009). Pire, on assiste à une dégradation des sols suite à leur mise en culture (Coulibaly et al., 2012 ; Koulibaly et al., 2017 ; Amonmide et al., 2019). Aussi, le maintien de la fertilité des sols cultivés constitue-t-il un des défis majeurs que les pays concernés devraient relever pour assurer la durabilité de la production agricole en vue de la sécurité alimentaire des populations.

Pour faire face aux contraintes pédologiques, la recherche, à travers des essais de durée plus ou moins longue menés aussi bien en station qu'en milieu paysan, a développé des technologies capables d'entretenir voire d'améliorer la fertilité des sols. Mason et al. (2014) ont fait la synthèse de résultats obtenus dans la région semi-aride de l'Afrique de l'Ouest. En effet, plusieurs options de gestion de la fertilité des sols basées, entre autres, sur la rotation culturale (Koulibaly et al., 2016), l'introduction des légumineuses dans les rotations (Bado et al., 2012), la gestion des résidus de cultures (Koulibaly et al., 2010 ; Ouattara et al., 2011 ; Abdou et al., 2016; Koulibaly et al., 2017), l'utilisation combinée des engrais et de la fumure organique (Koulibaly et al., 2015 ; Akanza et al., 2016) et les aménagements antiérosifs (Zougmoré, 2003), ont montré leur efficacité dans l'amélioration de la productivité des cultures et des sols.
Cependant, les exploitations agricoles de la zone sont confrontées à des contraintes techniques et socioéconomiques qui limitent l'adoption des innovations proposées par la recherche (Yabi et al., 2016; Kohio et al., 2017). Par conséquent, les technologies sont diversement et/ou le plus souvent insuffisamment mises en œuvre par les producteurs (Hauchart, 2006; Blanchard, 2010 ; Yabi et al., 2016 ; Kohio et al., 2017). En effet, sur la base de leurs expériences et/ou avec l'appui des partenaires techniques, les producteurs développent leurs propres pratiques de gestion des sols en relation avec leurs conditions socio-économiques. Il parait alors important de connaitre les pratiques agricoles des producteurs et leurs effets sur les caractéristiques des sols afin d'aider à développer des innovations adaptées permettant une utilisation durable des sols. Malheureusement très peu d'études ont évalué l'impact des systèmes agricoles paysans sur la fertilité des sols. La plupart des données disponibles sur le sujet provient de dispositifs expérimentaux mis en place en milieu paysan en collaboration avec les producteurs.

Le présent travail, effectué dans la zone cotonnière à l'Ouest du Burkina Faso, considérée comme «le grenier» du pays, s'inscrit dans ce contexte avec pour objectif d'évaluer les effets de différentes pratiques de fertilisation mises en œuvre dans les systèmes de culture sur les caractéristiques d'un sol ferrugineux tropical lessivé. 


\section{MATERIEL ET METHODES \\ Caractéristiques des situations étudiées}

L'étude a eu lieu dans le terroir de Kadomba situé à 50 kilomètres au Nord de Bobo-Dioulasso, entre $11^{\circ} 23$ et $11^{\circ} 41$ de Latitude Nord et, $3^{\circ} 45$ et $4^{\circ} 8$ de Longitude Ouest. Le climat est de type Sud-soudanien (Fontes et Guinko, 1995) avec une pluviométrie annuelle comprise entre 900 et $1200 \mathrm{~mm}$. Le terroir se trouve dans la zone cotonnière ouest caractérisée par des systèmes de culture céréaliers à base de coton (MARA, 1997).

Le sol étudié est un sol ferrugineux tropical lessivé, de couleur brune en surface à brun rougeâtre en profondeur. Sa texture est sableuse en surface à argileuse en profondeur. Les caractéristiques analytiques sont données dans le Tableau 1.

\section{Caractérisation des systèmes de fertilisation}

L'étude a été réalisée avec un échantillon de vingt-deux (22) parcelles choisies en fonction de la pratique ou non de la fumure organique, après une enquête préliminaire ; la moitié des parcelles a reçu des apports organiques et l'autre moitié n'en a bénéficié. Le passé cultural, en particulier le système de fertilisation de chaque parcelle a été caractérisé sur une période de six (6) ans (2010-2015) à travers des enquêtes auprès des producteurs ainsi que des observations sur le terrain. Les enquêtes ont permis d'estimer les quantités d'engrais et de fumier apportées sur chaque parcelle durant les six (6) années. Les quantités d'engrais ont été estimées en nombre de sacs ou de boîtes puis ramenées en $\mathrm{kg}$ en raison de $50 \mathrm{~kg}$ le sac et de $2 \mathrm{~kg}$ la boîte utilisée dans la zone. Concernant le fumier, les quantités apportées par transport au champ ont été estimées en nombre de charretées puis ramenées en $\mathrm{kg}$ en raison de $200 \mathrm{~kg}$ de matière sèche la charretée selon Waneukem (1996). Par contre pour le parcage des animaux, les quantités de fumier apportées par cette pratique ont été estimées à 10 tonnes par hectare et par an (Milleville, 1982).

\section{Détermination des paramètres chimiques}

Dans chacune des vingt-deux (22) parcelles, un échantillon composite de la couche $0-20 \mathrm{~cm}$ a été constitué à partir de 25 échantillons élémentaires pour des analyses au laboratoire. Les paramètres suivants ont été analysés sur ces échantillons : carbone total, azote total, $\mathrm{pH}$ eau, phosphore assimilable, $\mathrm{CEC}$ et bases échangeables. Les analyses ont été faites selon les méthodes en vigueur au laboratoire d'analyses du BUNASOLS (1987).

\section{Analyse des données}

Après les déterminations analytiques, les résultats ont été analysés en fonction des différentes pratiques de fertilisation qui ont été identifiées et caractérisées à l'issu des enquêtes. L'analyse statistique des données a été effectuée à l'aide du logiciel XLSTAT, version 2016. Le test de Newman Keuls a permis de comparer les moyennes au seuil de $5 \%$ lorsque l'analyse de variance indique des différences entre les pratiques.

Tableau 1 : Caractéristiques chimiques du sol ferrugineux tropical lessivé sous végétation naturelle.

\begin{tabular}{|c|c|c|c|c|c|c|c|c|c|c|c|c|c|c|}
\hline \multirow[t]{2}{*}{$\begin{array}{l}\text { Profondeur } \\
\text { (cm) }\end{array}$} & \multicolumn{3}{|c|}{ Granulométrie } & \multicolumn{2}{|c|}{$\begin{array}{l}\text { Matière } \\
\text { organique }\end{array}$} & \multicolumn{4}{|c|}{$\begin{array}{l}\text { CEC et Bases échangeables } \\
\left(\text { Cmol.kg }^{-1} \text { de sol) }\right.\end{array}$} & \multicolumn{3}{|c|}{ pH eau } & \multirow{2}{*}{\multicolumn{2}{|c|}{$\begin{array}{l}\text { Phosphore } \\
\text { assimilable } \\
\text { (mg.kg }{ }^{-1} \\
\text { de sol) }\end{array}$}} \\
\hline & $\begin{array}{l}\text { Argile } \\
(\%)\end{array}$ & $\begin{array}{l}\text { Limon } \\
(\%)\end{array}$ & $\begin{array}{l}\text { Sable } \\
(\%)\end{array}$ & $\begin{array}{l}\text { MO } \\
(\%)\end{array}$ & $\begin{array}{l}\mathrm{Ct} \\
(\%)\end{array}$ & $\begin{array}{l}\mathrm{Nt} \\
(\%)\end{array}$ & $\mathrm{Ca}^{2+}$ & $\mathrm{Mg}^{2+}$ & $\mathbf{K}^{+}$ & $\mathbf{N a}^{+}$ & SB & CEC & & \\
\hline $0-20$ & 7,8 & 33,8 & 58,8 & 1,16 & 0,67 & 0,07 & 1,24 & 0,58 & 0,21 & 0,05 & 2,08 & 5,15 & 5,8 & 2,15 \\
\hline $20-40$ & 19,6 & 33,3 & 47,1 & 1,03 & 0,60 & 0,08 & 1,51 & 0,82 & 0,08 & 0,06 & 2,47 & 4,45 & 5,4 & 1,15 \\
\hline $40-55$ & 35,3 & 37,3 & 27,3 & 1,17 & 0,68 & 0,12 & 1,11 & 0,76 & 0,08 & 0,05 & 2,00 & 4,25 & 5,2 & 0,72 \\
\hline $55-120$ & 39,2 & 23,5 & 37,3 & 1,02 & 0,59 & 0,16 & 1,15 & 0,57 & 0,08 & 0,06 & 1,86 & 4,56 & 5,4 & 0,29 \\
\hline
\end{tabular}

$\mathrm{Ct}=$ carbone organique total, $\mathrm{MO}=$ matière organique, $\mathrm{Nt}=$ azote total, $\mathrm{CEC}=$ capacité d'échange cationique, $\mathrm{SB}=$ Somme des bases échangeables. 


\section{RESULTATS \\ Principales pratiques de fertilisation}

Les caractéristiques des systèmes de cultures mis en œuvre dans les 22 parcelles étudiées sont présentées dans les Tableaux 2 et 3. La plupart des systèmes $(77,3 \%)$ se caractérise par la culture de céréales (maïs et sorgho) en rotation avec le cotonnier, les légumineuses (arachide) et/ou la jachère. En plus de ces systèmes, il y a des systèmes de culture continue de céréales souvent en rotation entre elles $(22,7 \%)$.

La pratique de la jachère a concerné 40,9\% des parcelles étudiées. En effet, durant la succession culturale des 6 années considérées, la durée de la jachère a été de un, trois, quatre ou de 5 ans. A l'exception de la jachère d'une année intervenue entre deux cultures, toutes les autres jachères ont été enregistrées au début de période considérée. En d'autres termes, il s'agit de parcelles remises en culture depuis un certain temps après une période de jachère.

Les pratiques de fertilisation se caractérisent par l'apport ou non de fumier et d'engrais NPK et urée. Les quantités apportées sont présentées dans le Tableau 3. En effet, la moitié des parcelles a reçu, de 2010 à 2015, au moins un apport annuel de fumier soit par parcage des animaux ou par transport dans les champs à l'aide de charrettes à traction bovine. Les quantités apportées ont été estimées en moyenne à 9019 $\mathrm{kg} / \mathrm{ha}$ avec des valeurs variant entre 2500 et $13500 \mathrm{~kg} / \mathrm{ha}$. Concernant la fumure minérale, seules les parcelles sous culture de maïs ou de cotonnier, ont reçu un apport d'engrais (NPK et/ou urée). Ainsi, pour les six (6) dernières de successions culturales, $22,7 \%$ des parcelles n'ont reçu aucun apport de fumure minérale, $40,9 \%$ n'en ont bénéficié qu'une seule fois alors que les autres $(36,4 \%)$ ont été fertilisées au moins deux fois. Les quantités d'engrais apportées ont été estimées en moyenne à $130,80 \mathrm{~kg} / \mathrm{ha}$ de $\mathrm{NPK}$ et $33,95 \mathrm{~kg} / \mathrm{ha}$ d'urée pour le cotonnier contre $125,56 \mathrm{~kg} / \mathrm{ha}$ de NPK et $56,67 \mathrm{~kg}$ d'urée pour le maïs. Après la récolte et en absence de parcage d'animaux, la quasi-totalité des résidus sont exportés des champs.
Effet des pratiques de fertilisation sur les caractéristiques chimiques du sol ferrugineux tropical lessivé

Les caractéristiques chimiques du sol déterminées dans les différents systèmes de culture sont données dans le Tableau 4. Selon les normes d'interprétation du BUNASOLS (1987), le niveau des teneurs en matière organique varie de très faible $(0,93 \%)$ à moyen (2,36\%). Celui de l'azote total, compris entre 0,049 à 0,097\%, est faible. La capacité d'échange cationique (CEC) varie de très faible $\left(3,51 \mathrm{Cmol} . \mathrm{kg}^{-1} \mathrm{de}\right.$ sol) à faible (8,23 Cmol. $\mathrm{kg}^{-1}$ de sol). La somme des bases échangeables est faible avec des valeurs variant entre 2,54 et $5,49 \mathrm{Cmol} . \mathrm{kg}^{-1}$ de sol. Concernant la réaction du sol, les valeurs du $\mathrm{pH}$ vont de fortement acide $(5,3)$ à neutre $(6,8)$. Les teneurs en phosphore assimilable sont très faibles avec des valeurs variant de 2,65 et 8,94 mg. $\mathrm{kg}^{-1}$ de sol.

\section{Effet de la pratique de la fumure organique}

Dans le Tableau 5 sont comparées les caractéristiques du sol dans les systèmes de culture pratiquant la fumure organique à celles du sol sous systèmes de culture sans apport de fumure organique. Il ressort que la teneur en matière organique, la $\mathrm{CEC}$, la somme des bases échangeables et le $\mathrm{pH}$ du sol sont significativement $(\mathrm{P}<5 \%)$ plus élevés dans les parcelles ayant bénéficié de la pratique de fumure organique par rapport aux parcelles où cette pratique a été absente. Les augmentations par rapport au sol non fumé sont de $+0,32 \%$ pour la teneur en matière organique, de $+1,08 \mathrm{Cmol} . \mathrm{kg}^{-1}$ de sol pour la CEC, de $+0,91$ Cmol. $\mathrm{kg}^{-1}$ de sol pour la zone des bases échangeables et de $+0,30$ unité pour le $\mathrm{pH}$ eau. Par contre, les teneurs en azote total et en phosphore assimilable n'ont pas montré une différence significative entre les parcelles avec toutefois des valeurs moyennes relativement plus élevées pour celles ayant bénéficié de la fumure organique.

\section{Effet de la pratique de la fumure minérale}

La comparaison des caractéristiques du sol en fonction de la fréquence des apports de fumure minérale (NPK+urée) durant la succession culturale des six (6) dernières années (2010 à 2015) ne montre pas de 
différence significative (Tableau 6). Mais on peut observer que les valeurs de la teneur en matière organique, de la $\mathrm{CEC}$ et du $\mathrm{pH}$ ont tendance à diminuer dans les parcelles où les apports de fumure minérale sont répétés par rapport aux parcelles sans apport. Le contraire est observé concernant les teneurs en azote total, en phosphore assimilable et la somme des bases échangeables dont les valeurs moyennes sont légèrement plus élevées avec les apports répétés.

\section{Effet de la pratique de la jachère}

Le Tableau 7 montre que les caractéristiques du sol dans les parcelles ayant bénéficié d'une période de jachère ne sont pas significativement différentes de celles du sol cultivé sans jachère. Cependant les données indiquent une tendance à l'augmentation des teneurs en matière organique, azote total et en phosphore avec la pratique jachère. Par contre, la CEC, la somme des bases échangeables et le $\mathrm{pH}$ eau tendent à diminuer dans les parcelles avec jachère.

Tableau 2: Caractéristiques des systèmes de culture.

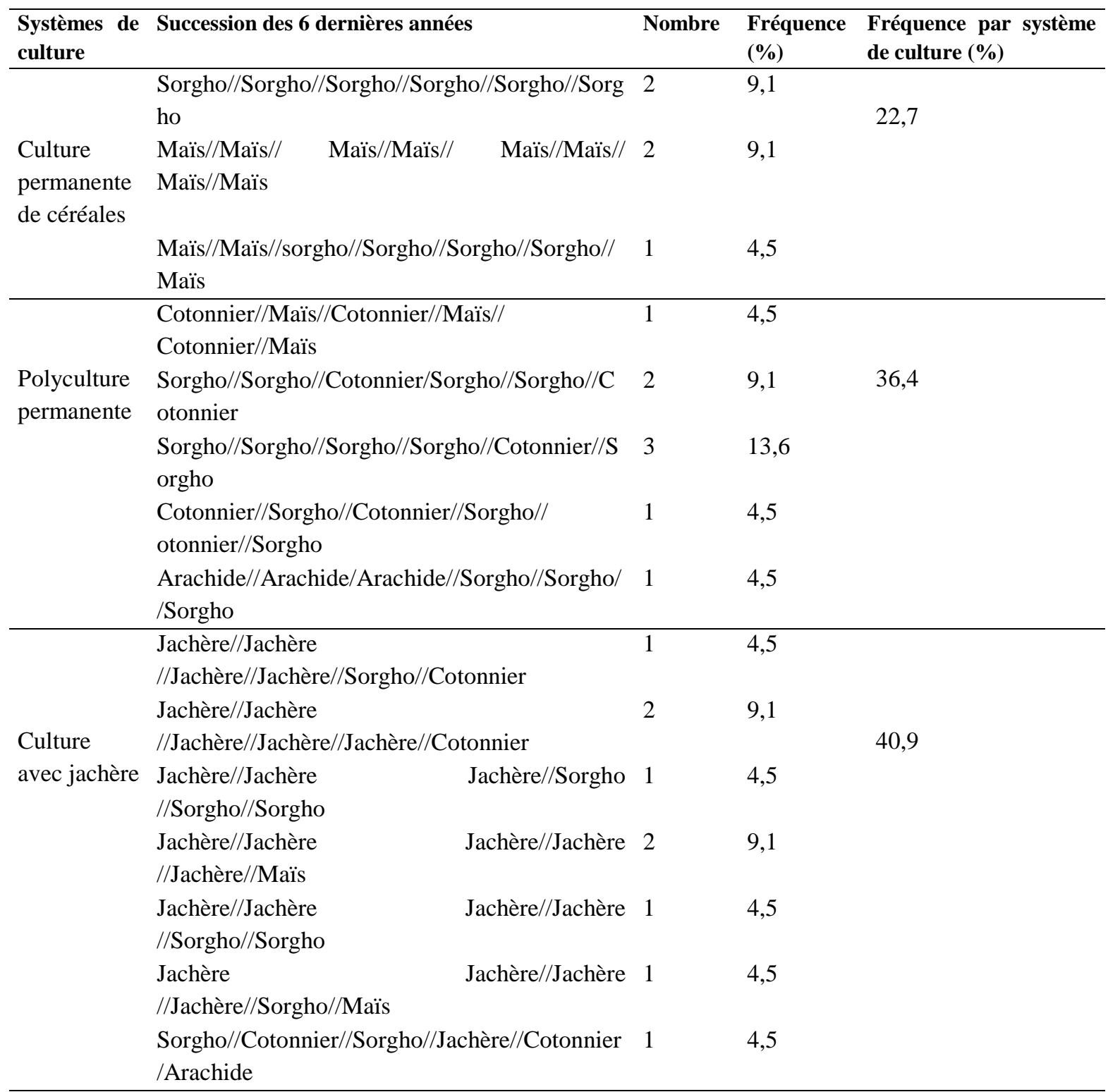


Tableau 3: Age de mise en culture des parcelles et quantités des fertilisants apportées par spéculation durant les six dernières années.

\begin{tabular}{|c|c|c|c|c|c|c|}
\hline & \multirow[t]{3}{*}{$\begin{array}{l}\text { Age de mise en } \\
\text { culture (années) }\end{array}$} & \multirow[t]{3}{*}{$\begin{array}{l}\text { Quantité de fumure } \\
\text { organique apportée }(\mathrm{kg} / \mathrm{ha})\end{array}$} & \multicolumn{4}{|c|}{$\begin{array}{l}\text { Quantités de fumure } \\
\text { minérale apportée }\end{array}$} \\
\hline & & & \multicolumn{2}{|c|}{ Cotonnier } & \multicolumn{2}{|l|}{ Maïs } \\
\hline & & & $\begin{array}{l}\text { NPK } \\
\text { (kg/ha) }\end{array}$ & $\begin{array}{l}\begin{array}{l}\text { Urée } \\
\text { (kg/ha) }\end{array} \\
\end{array}$ & $\begin{array}{l}\text { NPK } \\
\text { (kg/ha) }\end{array}$ & $\begin{array}{l}\begin{array}{l}\text { Urée } \\
\text { (kg/ha) }\end{array} \\
\end{array}$ \\
\hline $\begin{array}{l}\text { Valeur } \\
\text { Moyenne }\end{array}$ & 22 & 9019 & 130,8 & 33,95 & 125,56 & 56,67 \\
\hline $\begin{array}{l}\text { Valeurs } \\
\text { extrêmes }\end{array}$ & $15-31$ & $2500-13500$ & $50-200$ & $0-100$ & $50-200$ & $50-100$ \\
\hline
\end{tabular}

Tableau 4 : Caractéristiques chimiques de l'horizon $0-20 \mathrm{~cm}$ du sol ferrugineux tropical lessivé toutes situations culturales confondues.

\begin{tabular}{|c|c|c|c|c|c|c|c|}
\hline & \multicolumn{7}{|c|}{ Caractéristiques du sol } \\
\hline & $\begin{array}{l}\mathrm{Ct} \\
(\%)\end{array}$ & $\begin{array}{l}\text { MO } \\
(\%)\end{array}$ & $\begin{array}{l}\mathrm{Nt} \\
(\%)\end{array}$ & $\begin{array}{l}\text { CEC } \\
\left(\mathrm{Cmol.kg}^{-1}\right. \\
\text { de sol })\end{array}$ & $\begin{array}{l}\text { SB } \\
\text { (Cmol.kg- } \\
{ }^{1} \text { de sol) }\end{array}$ & pH eau & $\begin{array}{l}\text { Phosphore } \\
\text { assimilable } \\
\left(\mathrm{mg.kg}^{-1} \text { de sol) }\right.\end{array}$ \\
\hline $\begin{array}{l}\text { Valeurs } \\
\text { moyennes }\end{array}$ & 0,92 & 1,59 & 0,067 & 4,89 & 3,45 & 6,24 & 4,93 \\
\hline Ecart type & 0,20 & 0,35 & 0,011 & 1,19 & 0,81 & 0,35 & 1,90 \\
\hline Valeurs extrêmes & $\begin{array}{l}0,54- \\
1,37\end{array}$ & $\begin{array}{l}0,93- \\
2,36\end{array}$ & $\begin{array}{l}0,049- \\
0,097\end{array}$ & $3,51-8,23$ & $2,54-5,49$ & $\begin{array}{l}5,30- \\
6,80\end{array}$ & $2,65-8,94$ \\
\hline
\end{tabular}

$\mathrm{Ct}=$ carbone organique total, $\mathrm{MO}=$ matière organique, $\mathrm{Nt}=$ azote total, $\mathrm{CEC}=$ capacité d'échange cationique, $\mathrm{SB}=\mathrm{Somme}$ des bases échangeables.

Tableau 5: Caractéristiques chimiques de l'horizon 0-20 cm du sol ferrugineux tropical lessivé en fonction des pratiques de fumure organique.

\begin{tabular}{|c|c|c|c|c|c|c|c|}
\hline \multirow[b]{2}{*}{$\begin{array}{l}\text { Pratique } \\
\text { fumure } \\
\text { organique }\end{array}$} & \multicolumn{7}{|c|}{ Caractéristiques du sol } \\
\hline & $\begin{array}{l}\mathrm{Ct} \\
(\%)\end{array}$ & $\begin{array}{l}\text { MO } \\
(\%)\end{array}$ & $\begin{array}{l}\mathrm{Nt} \\
(\%)\end{array}$ & $\begin{array}{l}\text { CEC } \\
\left(\mathrm{Cmol} . \mathrm{kg}^{-1}\right. \\
\text { de sol })\end{array}$ & $\begin{array}{l}\text { SB } \\
\left(\mathrm{Cmol}_{\mathrm{kg}} \mathrm{kg}^{-1}\right. \\
\text { de sol })\end{array}$ & $\mathrm{pH}$ eau & $\begin{array}{l}\text { Phosphore } \\
\text { assimilable } \\
\left(\mathrm{mg} \cdot \mathrm{kg}^{-1} \text { de sol) }\right.\end{array}$ \\
\hline SFO $(n=11)$ & $0,83 a$ & $1,43 a$ & 0,063 & $4,35 \mathrm{a}$ & $2,99 a$ & $6,10 a$ & 4,36 \\
\hline $\mathrm{AFO}(\mathrm{n}=11)$ & $1,02 b$ & $1,75 b$ & 0,074 & $5,43 b$ & $3,90 \mathrm{~b}$ & $6,40 \mathrm{~b}$ & 5,56 \\
\hline Probabilité & 0,03 & 0,03 & 0,145 & 0,03 & 0,005 & 0,04 & 0,14 \\
\hline Signification & S & S & NS & $\mathrm{S}$ & $\mathrm{S}$ & S & NS \\
\hline
\end{tabular}


Tableau 6: Caractéristiques chimiques de l'horizon $0-20 \mathrm{~cm}$ du sol ferrugineux tropical lessivé en fonction des pratiques de fumure minérale.

\begin{tabular}{|c|c|c|c|c|c|c|c|c|}
\hline \multirow[b]{2}{*}{$\begin{array}{l}\text { Pratique } \\
\text { fumure } \\
\text { organique }\end{array}$} & \multirow[b]{2}{*}{ de } & \multicolumn{7}{|c|}{ Caractéristiques du sol } \\
\hline & & $\begin{array}{l}\mathrm{Ct} \\
(\%)\end{array}$ & $\begin{array}{l}\text { MO } \\
(\%)\end{array}$ & $\begin{array}{l}\text { Nt } \\
(\%)\end{array}$ & $\begin{array}{l}\text { CEC } \\
\left(\text { Cmol.kg }^{-1}\right. \\
\text { de sol })\end{array}$ & $\begin{array}{l}\text { SB } \\
\left(\mathrm{Cmol} \mathrm{kg}^{-1} \mathrm{de}\right. \\
\text { sol })\end{array}$ & $\begin{array}{l}\text { pH } \\
\text { eau }\end{array}$ & $\begin{array}{l}\text { Phosphore } \\
\text { assimilable } \\
\text { (mg.kg-1 de sol) }\end{array}$ \\
\hline SFm $(n=5)$ & & 0,93 & 1,60 & 0,063 & 5,27 & 3,40 & 6,40 & 3,62 \\
\hline $\operatorname{AFm1}(n=9)$ & & 0,97 & 1,67 & 0,067 & 4,60 & 3,39 & 6,19 & 4,92 \\
\hline $\operatorname{AFm} 2(n=8)$ & & 0,86 & 1,48 & 0,068 & 4,97 & 3,54 & 6,19 & 5,87 \\
\hline Probabilité & & 0,55 & 0,53 & 0,77 & 0,62 & 0,93 & 0,52 & 0,13 \\
\hline Signification & & NS & NS & NS & NS & NS & NS & NS \\
\hline
\end{tabular}

$\mathrm{SFm}$ = sans apport de fumure minérale durant les six dernières 6 années de culture ; AFm1 = apport de fumure minérale une seule fois durant les six dernières années de culture; $\mathrm{AFm} 2=$ apport de fumure plus d'une fois durant les six dernières années de culture ; $\mathrm{n}=$ nombre de parcelles concernées $; \mathrm{NS}=$ non significatif $; \mathrm{Ct}=$ carbone organique total, $\mathrm{MO}=$ matière organique, $\mathrm{Nt}=$ azote total, $\mathrm{CEC}=$ capacité d'échange cationique, $\mathrm{SB}=$ Somme des bases échangeables.

Tableau 7 : Caractéristiques chimiques de l'horizon 0-20 cm du sol ferrugineux tropical lessivé en fonction des pratiques de la jachère.

\begin{tabular}{|c|c|c|c|c|c|c|c|}
\hline \multirow[b]{2}{*}{$\begin{array}{l}\text { Pratique de } \\
\text { fumure } \\
\text { organique }\end{array}$} & \multicolumn{7}{|c|}{ Caractéristiques du sol } \\
\hline & $\begin{array}{l}\text { Ct } \\
(\%)\end{array}$ & $\begin{array}{l}\text { MO } \\
(\%)\end{array}$ & $\begin{array}{l}\text { Nt } \\
(\%)\end{array}$ & $\begin{array}{l}\text { CEC } \\
\left(\mathrm{Cmol.kg}^{-1}\right. \\
\text { de sol })\end{array}$ & $\begin{array}{l}\text { SB } \\
\left(\mathrm{Cmol.kg}^{-1}\right. \\
\text { de sol })\end{array}$ & $\begin{array}{l}\text { pH } \\
\text { eau }\end{array}$ & $\begin{array}{l}\text { Phosphore } \\
\text { assimilable } \\
\left(\mathrm{mg.kg}^{-1} \text { de sol) }\right.\end{array}$ \\
\hline SJa $(n=13)$ & 0,88 & 1,51 & 0,066 & 5,07 & 3,50 & 6,29 & 4,36 \\
\hline $\mathrm{AJa}(\mathrm{n}=9)$ & 0,98 & 1,70 & 0,067 & 4,62 & 3,37 & 6,17 & 5,56 \\
\hline Probabilité & 0,25 & 0,24 & 0,83 & 0,40 & 0,71 & 0,45 & 0,14 \\
\hline Signification & NS & NS & NS & NS & NS & NS & NS \\
\hline
\end{tabular}

$\mathrm{SJa}=$ sans jachère durant les six dernières 6 années de culture ; AJa $=$ jachère au moins une fois durant les six dernières années de culture $; \mathrm{n}=$ nombre de parcelles concernées $; \mathrm{NS}=$ non significatif $; \mathrm{Ct}=$ carbone organique total, $\mathrm{MO}=$ matière organique, $\mathrm{Nt}=$ azote total, $\mathrm{CEC}=$ capacité d'échange cationique, $\mathrm{SB}=$ Somme des bases échangeables. 


\section{DISCUSSION}

Les résultats confirment le faible niveau de la plupart des caractéristiques chimiques du sol quel que soit le système de culture, comme cela a été rapporté par Sawadogo et al. (2008), Bationo (2009) et Amonmide et al. (2019). Les faibles valeurs des caractéristiques sont à mettre au compte de l'origine des sols, des conditions climatiques qui sont agressives et des systèmes de culture. En effet, l'origine lithologique et les conditions de la pédogénèse tropicale font de la plupart des sols ferrugineux, des sols à faible fertilité (Pallo et al., 2008 ; Bationo, 2009). De plus, ces sols sont mis en culture avec des systèmes de culture qui les exposent à l'érosion et/ou à l'appauvrissement (Bationo, 2009 ; Bacyé et Boro, 2011).

Cependant, on enregistre une amélioration significative du niveau de la plupart des caractéristiques chimiques analysées dans les systèmes de culture comportant des apports de fumure organique comparativement à ceux où cette pratique est absente. Ces résultats ne corroborent pas ceux de Pouya et al. (2013) qui, dans la même zone cotonnière, n'ont pas pu mettre en évidence un effet significatif des pratiques paysannes de la fumure organique sur les caractéristiques chimiques des sols. Cette divergence des résultats pourrait s'expliquer par le fait que les travaux de Pouya et al. (2013) n'ont pas porté sur un type de sol particulier. Nos résultats confirment par contre, ceux enregistrés lors d'essais réalisés en station (Koulibaly et al., 2010 et 2015 ; Akanza et al., 2016 ) et en milieu paysan (Sawadogo et al., 2008 ; Naïtormbaide, 2012). La pratique de la fumure organique par parcage des animaux sur le champ ou par apport de fumier transporté au champ constitue donc un moyen formidable d'entretien voire d'amélioration de la fertilité des sols. Cependant, selon Dugué (2002) et Tonneau et al. (2002), la pratique de la fumure organique au sein des exploitations agricoles, implique un transfert de fertilité basé sur l'utilisation des ressources organiques d'un grand espace (zone de culture, pâturage) au profit d'un espace plus réduit (portion du champ fumée). En effet, les apports organiques qui se font sous forme de fumier ou de compost, concernent une faible proportion des superficies cultivées. Blanchard (2010) a estimé les surfaces fumées entre 9 et $21 \%$ des superficies cultivées des exploitations agricoles au sud Mali. Si les quantités apportées sont assez appréciables dans les parties des champs qui en reçoivent, elles deviennent très insignifiantes lorsqu'on considère toutes les superficies exploitées (Blanchard, 2010 ; Bacyé et Boro, 2011). Cela pose le problème de l'utilisation des matières organiques transformées (fumier, compost) comme principale forme de restitution organiques dans les exploitations agricoles.

La pratique de la fumure minérale (NPK et urée), réservée uniquement aux cultures du cotonnier et du maïs, semble ne pas avoir un effet bien défini et significatif sur les caractéristiques du sol. L'apport d'engrais est considéré comme une pratique de gestion durable des sols dans la zone parce qu'il peut permettre d'accroître les restitutions organiques à travers une augmentation de la production de biomasse végétale (Vanlauwe and Giller, 2006 ; Bationo, 2009), en plus de son effet sur les rendements des cultures. Mais l'absence d'effet de la pratique de la fumure minérale pourrait s'expliquer par le fait que les quantités apportées sont relativement faibles. En effet, les quantités d'engrais apportés évaluées en moyenne à 164 et 182 $\mathrm{kg} / \mathrm{ha}$ de NPK + urée respectivement pour le cotonnier et le maïs, sont en deçà des doses recommandées qui sont de 200 et $300 \mathrm{~kg} / \mathrm{ha}$ respectivement (INERA, 2000). De plus, ces quantités ne sont apportées que sur les cultures de cotonnier et de maïs, c'est-à-dire une année sur deux ou trois. 
Les résultats révèlent également une contribution peu nette et non significative de la jachère sur les caractéristiques du sol. Koulibaly et al. (2014), ont aussi rapporté que 15 années de jachère n'ont pas permis d'améliorer les teneurs en carbone et azote total du sol par rapport à des systèmes pratiquant des labours mécanisés. L'absence d'effet de la pratique de la jachère pourrait s'expliquer par la faible durée des jachères et aussi par la présence quasiment généralisée des feux de brousse qui détruisent chaque année la végétation des jachères. De plus, les changements climatiques de ces dernières années, marqués par une baisse de la pluviosité, ne sont pas favorables au développement de la végétation dans les parcelles abandonnées.

\section{Conclusion}

L'étude a été conduite dans la zone cotonnière à l'Ouest du Burkina Faso en vue d'évaluer les effets des pratiques de fertilisation mises en œuvre dans les systèmes de culture sur les caractéristiques d'un sol ferrugineux tropical lessivé. Les résultats ont montré que les pratiques actuelles de gestion de la fertilité des sols au sein des exploitations, marquées par l'exportation de la quasi-totalité des résidus de récolte, la pratique de la jachère de courte durée, l'apport de faibles doses d'engrais NPK + urée uniquement sur le cotonnier et le maïs et, l'apport localisé de la fumure organique sur de faibles superficies, ne pourront pas maintenir durablement la productivité des sols. En effet, seule la pratique de la fumure organique apportant le fumier par parcage des animaux ou par transport au champ, a permis une amélioration significative de la plupart des caractéristiques chimiques du sol qui demeurent toutefois à des niveaux faibles, selon les normes disponibles dans la région. Cela confirme cependant le rôle central et incontournable de la fumure organique dans le maintien voire l'amélioration de la fertilité des sols. Aussi, le renforcement des restitutions organiques constitue-t-il un des enjeux de la gestion durable des sols dans cette zone. Mais les modes de restitutions actuels, basés essentiellement sur l'apport des matières organiques plus ou moins transformées (fumier et compost), ne pourraient permettre de fumer que de faibles portions par rapport à l'ensemble des superficies cultivées entrainant ainsi un transfert de fertilité au sein des exploitations. Aussi, la recherche de solution pour le renforcement des restitutions organiques devrait-elle s'orienter vers des systèmes de production, en particulier des systèmes de fertilisation, visant à accroitre la production de biomasse végétale et surtout les apports directs des résidus organiques au sol.

\section{CONFLIT D'INTERETS}

Les auteurs déclarent qu'il n'existe pas de conflit d'intérêts sur cet article.

\section{CONTRIBUTIONS DES AUTEURS}

Tous les auteurs ont participé à la conception de l'étude. BB a participé à la collecte des données de terrain, supervisé les analyses de laboratoire et traité les données. HSK et ASS ont apporté des conseils dans la collecte et le traitement de données. Tous les auteurs ont contribué à la rédaction du manuscrit et approuvé la version finale.

\section{REMERCIEMENTS}

Les auteurs remercient vivement l'Université Nazi BONI (UNB) et l'Institut de l'Environnement et de Recherches Agricoles (INERA) pour les infrastructures et les facilités administratives qui leur ont été accordées. Ils remercient également le Bureau national des sols (BUNASOLS) pour avoir facilité les analyses de sol. 


\section{REFERENCES}

Abdou G, Ewusi-Mensah N, Nouri M, Tetteh FM, Safo EY, Abaidoo RC. 2016. Nutrient release patterns of compost and its implication on crop yield under Sahelian conditions of Niger. Nutrient Cycling in Agroecosystems, 105(2): 117128.

DOI:

http://dx.doi.org/10.1007/s10705-0169779-9.

Akanza KP, Sanogo S, N'Da HA. 2016. Influence combinée des fumures organique et minérale sur la nutrition et le rendement du maïs: impact sur le diagnostic des carences du sol. Tropicultura, 34(2): 208-220.

Amonmide I, Dagbenonbakin G, Agbangba CE, Akponikpe P. 2019. Contribution à l'évaluation du niveau de fertilité des sols dans les systèmes de culture à base du coton au Bénin. Int. J. Biol. Chem. Sci., 13(3): 1846-1860. DOI: https://dx.doi.org/10.4314/ijbcs.v13i3.52

Bacyé B, Boro A. 2011. Study of organic matter flows in the Western cotton zone of Burkina Faso. Tropicultura, 29(3): 148-152.

Bado BV, Bationo A, Cescas M. 2012. Rôles des Légumineuses sur la Fertilité des Sols. Opportunites pour une Gestion Intégrée de la Fertilité des Sols. Editions universitaires européennes : Berlin.

Bationo A. 2009. Constraints and new opportunities for achieving a green revolution in Sub-Saharan Africa through Integrated Soil Fertility Management. The Proceedings of the International Plant Nutrition Colloquium XVI, UC Davis. p6.

Blanchard M. 2010. Gestion de la fertilité des sols et rôle du troupeau dans les systèmes coton-céréales-élevage au Mali-Sud, Savoirs techniques locaux et pratiques d'intégration agriculture élevage. Thèse de Doctorat, Université Paris-Est, Créteil Val de Marne, p. 298.

BUNASOLS. 1987. Méthodes d'analyse physique et chimique des sols, des eaux et des plantes. Documentation techniques $\mathrm{n}^{\circ}$ 3, BUNASOLS, Ouagadougou, p.159.

Coulibaly K, Vall E, Autfray P, Nacro HB, Sedogo MP. 2012. Effets de la culture permanente coton-maïs sur l'évolution d'indicateurs de fertilité des sols de l'Ouest du Burkina Faso. Int. J. Biol. Chem. Sci., 6(3): 1069-1080. DOI: http://dx.doi.org/10.4314/ijbcs.v6i3.13

Dugué P. 2002. Flux de biomasse et gestion de la fertilité à l'échelle des terroirs : étude de cas au Nord-Cameroun et essai de généralisation aux zones de savane d'Afrique sub-saharienne. In Fertilité et Relations Agriculture-Elevage en Zone de Savane : Actes de l'Atelier sur les Flux de Biomasse et la Gestion de la Fertilité à l'Echelle des Terroirs, 5-6 mai 1998, Dugué P (ed). CIRAD: Montpellier; 27-59.

Fontes J, Guinko S. 1995. Carte de la végétation et de l'occupation du sol du Burkina Faso: Notice explicative. Université de Ouagadougou, Ouagadougou. p. 67.

Hauchart V. 2006. Le coton dans le Mouhoun (Burkina Faso), un facteur de modernisation agricole : perspectives de développement? Cahiers Agricultures, 15(3): 285-291.

IN.E.R.A. 2000. Bilan de 10 années de recherche 1988-1998. MESSRS/CNRST, Ouagadougou. p.115.

Kohio EN, Touré AG, Sedogo MP, Ambouta J-MK. 2017. Contraintes à l'adoption des bonnes pratiques de Gestion Durable des Terres dans les zones soudaniennes et soudano-sahéliennes du Burkina Faso. Int. J. Biol. Chem. Sci., 11(6): $2982-$ 2989.

DOI: https://dx.doi.org/10.4314/ijbcs.v11i6.34 
Koulibaly B, Dakuo D, Ouattara A, Traoré O, Lompo F, Zombré NP, YaoKouamé A. 2015. Effets de l'association du compost et de la fumure minérale sur la productivité d'un système de culture à base de cotonnier et de maïs au Burkina Faso. Tropicultura, 33(2): 125-134.

Koulibaly B, Dakuo D, Traoré K, Ouattara A, Ouattara K, Traoré O, 2016. Soil tillage practices and crops rotations effects on yields and chemical properties of a lixisol in Burkina Faso. Journal of Applied Biosciences, 106: 10320-10332. DOI:

http://dx.doi.org/10.4314/jab.v106i1.12

Koulibaly B, Dakuo D, Traoré O, Ouattara K, Lompo F. 2017. Long-term effects of crops residues management on soil chemical properties and yields in cotton - maize - sorghum rotation system in Burkina Faso. Journal of Agriculture and Ecology Research, 10(2): 1-11. DOI: http://dx.doi.org/10.9734/JAERI/2017/3 1178.

Koulibaly B, Traoré O, Dakuo D, Zombré NP, Bondé D. 2010. Effets de la gestion des résidus de récolte sur les rendements et les bilans culturaux d'une rotation cotonnier-maïs-sorgho au Burkina Faso. Tropicultura, 28(3): 184-189.

Koulibaly B, Traoré O, Dakuo D, Lalsaga R, Lompo F, Zombré PN. 2014. Acidification des sols ferrugineux et ferrallitiques dans les systèmes de production cotonnière au Burkina Faso. Int. J. Biol. Chem. Sci., 8(6): 2879-2890. DOI:

http://dx.doi.org/10.4314/ijbcs.v8i6.44

MARA. 1997. Etude sur la typologie des exploitations agricoles familiales et adoption d'une nouvelle stratégie agricole. Rapport provisoire, MARA, Ouagadougou. p.230.

Mason SC, Ouattara K, Taonda SJB, Palé S, Sohoro A, Kaboré D. 2014. Soil and cropping system research in semiarid West Africa as related to the potential for conservation agriculture. International Journal of Agricultural Sustainability, 13(2).

DOI : http://dx.doi.org/10.1080/14735903.2014 .945319.

Milleville P. 1982. Etude d'un système de production agro-pastoral sahélien de Haute-Volta. $1^{\text {ère }}$ partie : Le système de culture. Rapport ORSTOM, Ouagadougou, p.66.

Naïtormbaide M. 2012. Incidence des modes de gestion des fumures et des résidus de récolte sur la productivité des sols dans les savanes du Tchad. Thèse de Doctorat, Université polytechnique de BoboDioulasso, Bobo-Dioulasso. p.171.

Ouattara K, Nyberg G, Ouattara B, Sedogo MP, Malmer A. 2011. Performances of Cotton-Maize rotation system as affected by ploughing frequency and soil fertility management in Burkina Faso A. In Innovations as Key to the Green Revolution in Africa, Bationo A et al. (eds). Springer Netherlands : Netherlands.

Pallo FJP, Sawadogo N, Sawadogo L, Sedogo PM. 2008. Statut de la matière organique des sols dans la zone sud-soudanienne au Burkina Faso. Biotechnol. Agron. Soc. Environ., 12(3) : 291-301.

Pouya MP, Bonzi M, Gnankambary Z, Traoré K, Ouédraogo JS, Somé AN, Sedogo MP. 2013. Pratiques actuelles de gestion de la fertilité des sols et leurs effets sur la production du cotonnier et sur le sol dans les exploitations cotonnières du Centre et de l'Ouest du Burkina Faso. Cah. Agric., 22(4): 282-292. http://dx.doi.org/10.1684/agr.2013.0643.

Sawadogo H, Bock L, Lacroix D, Zombré NP. 2008. Restauration des sols dégradés à l'aide du zaï et du compost dans le 
Yatenga (Burkina Faso). Biotechnol. Agron. Soc. Environ., 12(3): 279-290.

Tonneau JP, Sabourin E, Marçal da Silveira L, Sidersky P. 2002. Modélisation des flux de biomasse : une approche de la fertilité dans I' Agreste de la Paraiba (Brésil). Cahiers Agricultures, 11(2): 127-136.

Vanlauwe B., Giller KE. 2006. Popular myths around soil fertility management in subSaharan Africa. Agriculture, Ecosystems and Environment, 116: 34-46. DOI: http://dx.doi.org/10.1016/j.agee.2006.03. 016

Waneukem V. 1996. Appréciation de la fourniture d'azote par le sol. Cas des sols en maïs de la zone sud-soudanienne du Burkina Faso. Thèse de Doctorat. ENSA de Montpellier, Montpellier, p.246.
Yabi JA, Bachabi FX, Labiyi IA, Ode CA, Ayena RL.2016. Déterminants socioéconomiques de l'adoption des pratiques culturales de gestion de la fertilité des sols utilisées dans la commune de Ouaké au Nord-Ouest du Bénin. Int. J. Biol. Chem. Sci., 10(2): 779-792. DOI: http://dx.doi.org/10.4314/ijbcs.v10i2.27.

Zougmoré R. 2003. Effect of stone rows on soil chemical characteristics under continuous sorghum cropping in semiarid Burkina Faso. In Integrated Water and Nutrient Management for Sorghum Production in Semi-arid Burkina Faso, Leo Stroosnijder (ed). Wageningen University: Wageningen; 61-74. 